\title{
Development of a Novel Bidirectional Control Telecare System over a Wireless Sensor Network and the Internet
}

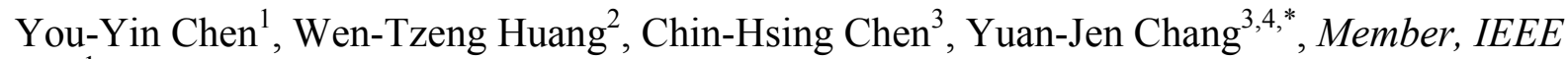 \\ ${ }^{1}$ Department of Electrical and Control Engineering, National Chiao Tung University \\ ${ }^{2}$ Department of Computer Science and Information Engineering \\ Minghsin University of Science and Technology \\ ${ }^{3}$ Department of Management Information Systems $/{ }^{4}$ Institute of Biomedical Engineering and \\ Material Science, Central Taiwan University of Science and Technology \\ *E-mail: ronchang@ctust.edu.tw
}

\begin{abstract}
The telecare system has become usable and accessible nowadays due to the development of information and communication technology. Its daily use has been made possible in long-term care through the use of wireless sensor networks. Unfortunately, the person in need of care may not be able to operate this sensor by him/herself. He/she is only able to turn this sensor on and off. Furthermore, he/she may be unconscious when an alert message is transferred. Against this backdrop, this paper therefore focuses on how the sensor can be automatically turned on to begin measuring physiological signals.

In this research, a bidirectional sensor controlling design for a wireless sensor network with a Web-based interface using the Internet was proposed. Rich internet application (RIA) technology was used to make a Web client-side user interface (UI) easier to use. Scalable vector graphics (SVG) technology, recommended by World Wide Web Consortium (W3C), was adopted to achieve dynamic charting of the browser. The combination of Ajax and SVG technology provides real-time interactivity and improves user experience on the use of the healthcare system. A complete system which includes the sensors, the wireless physiological detection network, the Web and database server, and the client side user interface was developed. To prove system reliability and accessibility, experiment and performance test of a real case were conducted.
\end{abstract}

Keywords: telecare, bidirectional, RIA, SVG

\section{Introduction}

According to the World Health Report [1], one of the most severe problems in a number of countries is the shortage of human resources which does not help answer the growing needs for health workers for longterm patient care. Chronic diseases and injuries make the situation even worse. Moreover, health workers need to learn new nursing skills using the more complex medical instruments. This leads to the increase in workload of each health worker. Due to this, it is therefore necessary to come up with a system with a friendly interface for users in order to increase medical quality. This study [2] indicates that an urgent alarm and communication system is necessary so patients can notify health workers immediately. Hence, a real-time bio-signal monitoring system needs to be developed.

It is a trend in the healthcare system to shift from a central, hospital-based system to a patient-centered system where patients become the manager and owner of their health information. However, the present healthcare system lacks interactive linkage of patients and health care workers.

In this study, we propose a solution for a home healthcare system in order to carry out functions that will meet future requirements. This system is database driven and has a Web-based interface. Online checking of patient's health status and real-time dynamic charting of physiological signals can be achieved with the use of Ajax and SVG technologies.. Moreover, the bandwidth of data transmission is reduced. An interactive interface designed by using rich internet application (RIA) improves its accessibility. The use of a wireless sensor network and a broadband network likewise increases the availability of this system. We also provide a novel method to allow users to control the remote sensor node bidirectionally through an easy-to-use Web-based interface via the Internet. 


\section{Related works}

Recently, Tura et al. [3] have focused on the implementation of a network for managing home-care activities. This network can measure blood oxygen saturation, heart rate, respiration rate, and the patient's quantity of movement. Measured data are stored in a multimedia card and then transmitted to a PC through bluetooth. The measured data are finally transmitted from the client side PC to the server side through the Internet. Since the wireless sensor network is comprised of a very large number of sensor nodes, a problem in management and querying is encountered. Generally, it is widely accepted that Web-based management should be used for querying and sensor network. To archive the Web-based management for wireless sensor network, Hwang et al. [4] implemented a sensor gateway comprised of two parts, namely, the Web server and Internet access module, and a sensor network access and management module. Multifunctions integrated in the sensor gateway provided Web-based access through the Internet with a public IP. Ferrari et al. [5] also provided the Bluetooth sensor network in which a Web server was built in the gateway. Operators can access data asynchronously through a Web-based interface, or synchronously through dynamic Web page refresh facility offered by HTML. The proposed architecture is shown in Fig. 1 $[4,5]$.

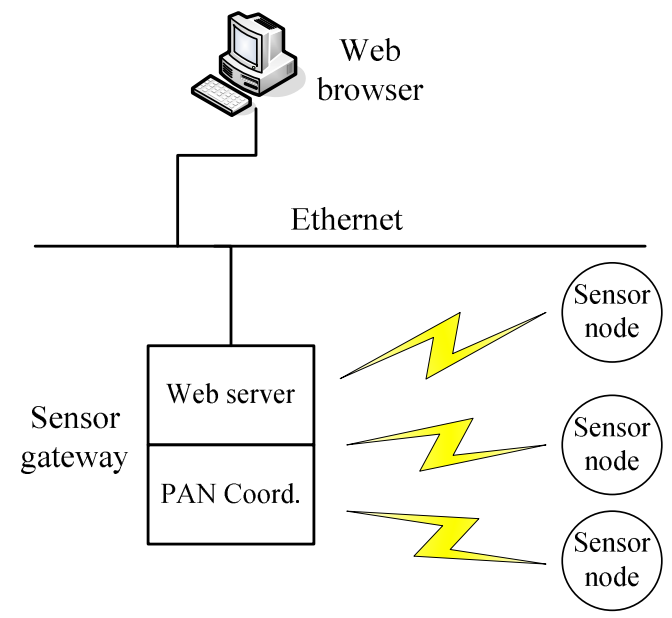

Figure 1. Web-enabled wireless sensor gateway $[4$, 5 ].

In several previous studies, enabling Internet access may cause some problems by embedding a Web server in the gateway. This design will increase the complexity and difficulty of wireless sensor gateway design and reduce the flexibility of the sensor gateway.
However, because of memory constraints, more powerful schemes will be restrained in the embedded Web server. If this embedded Web server is allowed to be accessed from the Internet, a public IP should be assigned to the Web server. This may possibly cause the embedded Web server to be unavailable under DoS attack risk.

\section{System architecture}

In this study, a proposed ZigBee-based networking architecture is shown in Fig. 2. An independent global Web apart from the gateway and the database server is constructed. The bio-signals detected by the sensors are transmitted to the gateway through the wireless sensor network. A Java program is developed to transmit bio-signal data from the sensor gateway to the remote database server. Client side users can access the bio-signal data through the Internet. The advantages of this architecture is that as compared to the previous one, only one public IP is required in the same group, and the gateway can hide behind the firewall to work safely. By using the database server, more control functions of the sensor can be developed. Moreover, bio-signal data from the multi-domain of wireless sensor gateways could be stored and accessed simultaneously through the database server. Interactive functions between the client side users and sensor nodes can be developed to improve user experience. In this study, a special table field is designed and combined with Ajax technology to attain real-time monitoring in the Web browsers. A detailed flow process will be explained in the next section.

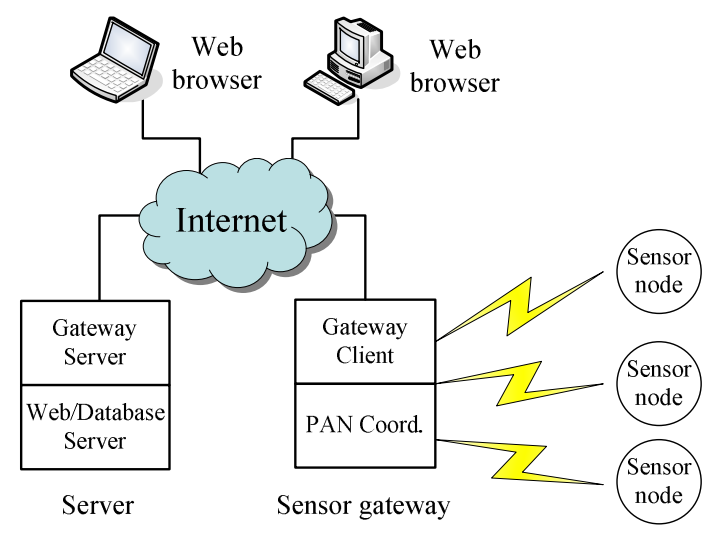

Figure 2. Proposed architecture in this study.

In this study, a Web server was built independently and apart from the sensor gateway. A gateway client module was embedded in the sensor gateway, and a gateway server module was embedded in the server. The gateway client module transmitted bio-signals, 
which were received from the sensor nodes, to the remote gateway server module. The gateway server module in turn stored the bio-signals into the database. A MySQL database system was set up to manage the raw data of bio-signals including Electrocardiogram (ECG) or percentage saturation of haemoglobin $\left(\mathrm{SpO}_{2}\right)$. A dynamic RIA Web page, which is comprised of XML, PHP, JavaScript, and Scalable Vector Graphics (SVG), was designed to show the dynamic physiological signal curve. Doctors can conveniently access the bio-signal data and manage the wireless sensor network via the Internet by just using the browser of whatever device, for example, a PC, notebook, or handheld device. Health workers can operate the healthcare monitoring system easily without extra training due to the easy-to-use RIA interface. Furthermore, the RIA Web page design improves user experience with Ajax technology.

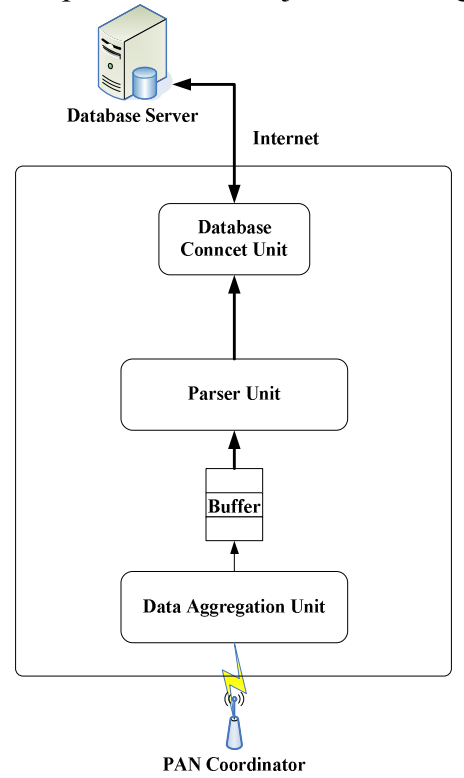

Figure 3. Flow diagram of the direct networking access method.

\subsection{Direct Networking Access}

Two database access methods were tested in this study. One was direct networking access, and the other was client-server access. In the direct networking access method, as shown in Fig. 3, the data aggregation unit received data from the sensor nodes. The database connection unit acquired bio-signal data by using the parser unit and then stored these data into a remote database server directly via the Internet.

\subsection{Client-Server Access}

In the client-server access method, as shown in Fig. 4 , a socket client unit was built. The bio-signal data acquired from the parser unit were not transmitted to the database directly. Instead, these data were first transmitted to the socket server unit via the Internet, and then were stored in the database server through the database connection unit.

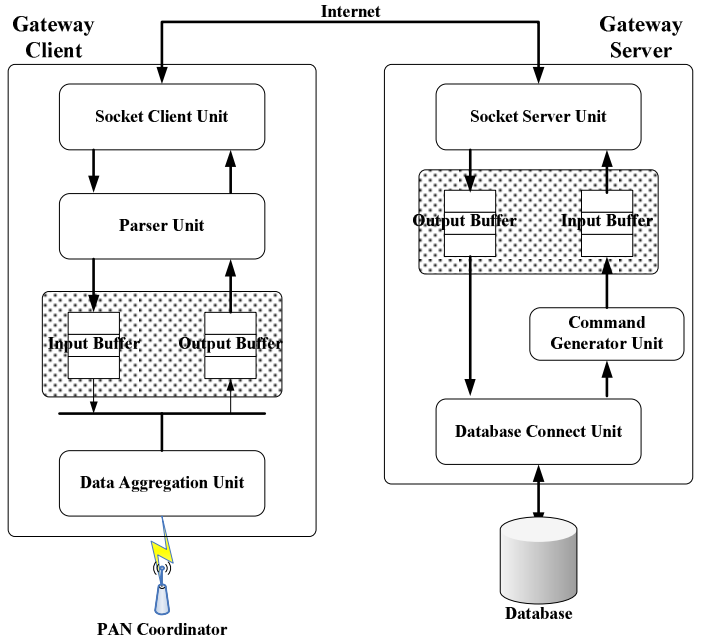

Figure 4. Flow diagram of the client-server access method.

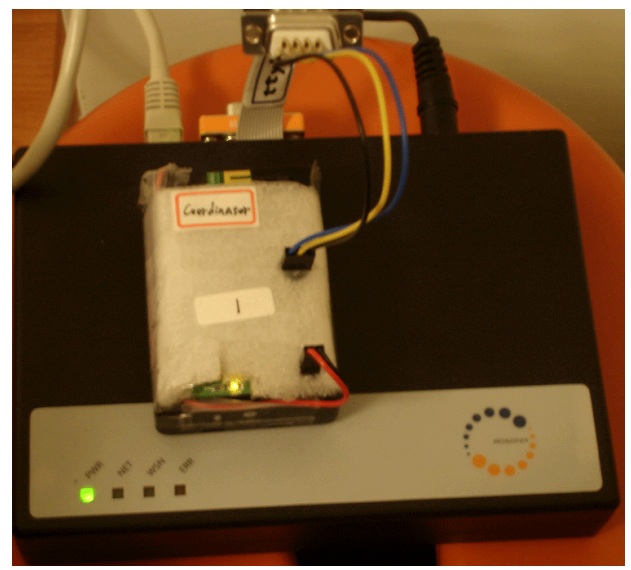

Figure 5. ZigBee gateway client module.

\subsection{Hardware Design of the Sensor Gateway}

An embedded platform provided by Rosonix Technology was used to develop the sensor gateway in this study. The platform uses the ARM9 processor of Samsung 2410 with 64M Byte NAND Flash and two $128 \mathrm{M}$ byte memory module on board. Embedded Linux 2.4 was installed, and $\mathrm{C} \backslash \mathrm{C}++$ language was used to develop the gateway client module. This sensor gateway was connected to the PAN coordinator through UART, and it connected to the Internet through the RJ-45 interface. A gateway server module 
was developed using Java language and communicated with JDBC API. Figure 5 shows the photograph of this sensor gateway module.

\section{Database Design}

In this study, in order to achieve bidirectional control between the client-side user and the sensor node, a special field such as a switch was designed in a sensor table. This field was used as register for sensor state. As shown in Fig. 6, a client-side user can use the browser to acquire the table field "switch" in the sensor table from the database server. An Ajax asynchronous function, sensor_state(), was designed to periodically select the database. In this study, this sensor_state() function was executed once per second. An appropriate retrieval period has been tested for better performance. While the gateway client obtained the information from the sensor node, a multithread function, sensor_swith(), immediately wrote the sensor data into the sensor table. Then the client-side user can reveal the state of the sensor almost at the same time through Ajax asynchronous function, sensor_state(). Similarly, if the client-side user wanted to

change the state of the sensor node, the user will only have to click the control button on the Web page. The Ajax asynchronous function, sensor_state(), will update the switch field in the sensor table immediately, and the gateway client will then obtain the state of the sensor from the switch field in the sensor table.

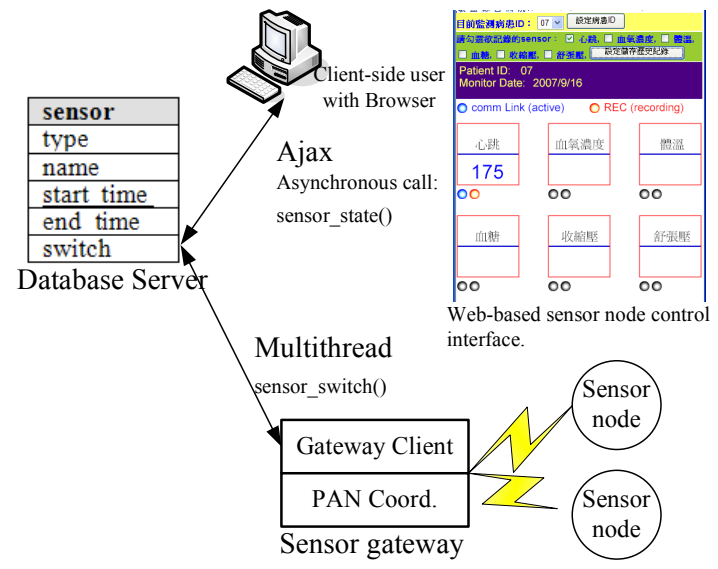

Figure 6. Bidirectional control between the clientside user and sensor node.

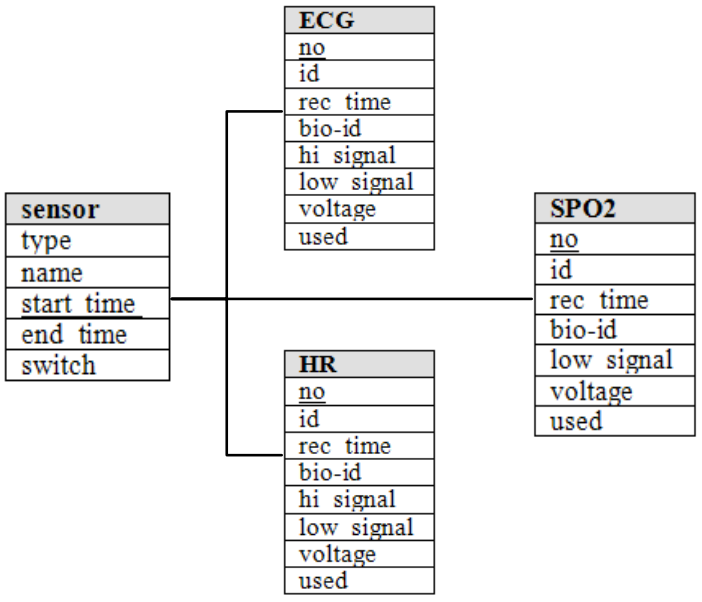

Figure 7. Database modeling.

According to the comparison between the present state and the new state, the gateway client will conduct the setting of the sensor node. Four sensor status recordings in the switch field were defined in this study: state " 0 " means sensor off, state " 1 " means asking the sensor to send data continuously, state " 2 " means asking the sensor to send data one time, and state " 3 " means asking the sensor to send data once per hour.

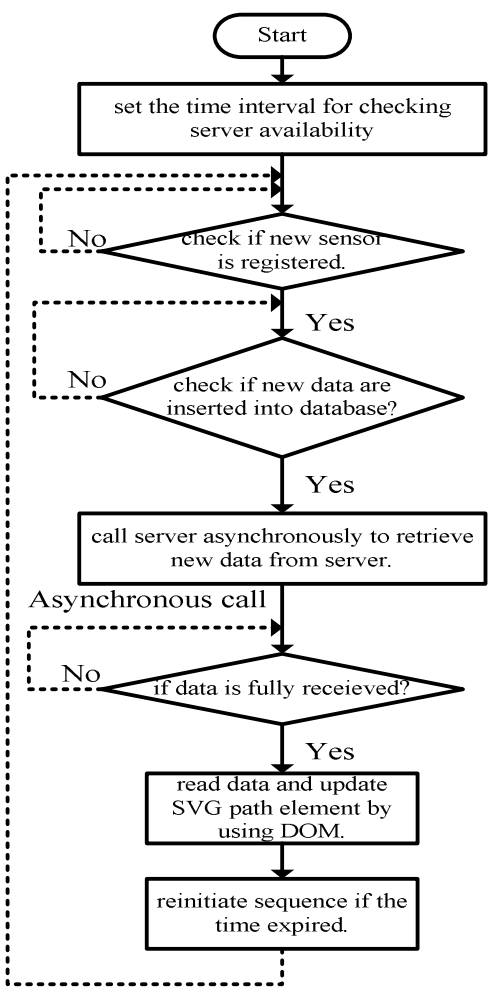

Figure. 8. Flowchart of the process of client side retrieving data from the database. 
The database is comprised of four tables, namely, ECG, heart rate, $\mathrm{SpO} 2$, and sensor status. The tables for $\mathrm{ECG}$, heart rate, $\mathrm{SpO}$, and plethysmographic pulse have the same fields which contain patient ID, signal ID, most significant byte of physiological value, least significant byte of physiological value, and used field. The "used" field records whether the physiological value has been read for charting in the user's browser. The sensor table is used to record the status of sensors. This table contains five fields, namely, type, name, start time, end time, and switch. The "type" field represents the types of physiological signals, which are ECG, heart rate, $\mathrm{SpO} 2$ value, $\mathrm{SpO} 2$ diagram, and status. The "switch" field represents the sensor status of power on or off. As the sensor power is turned on, a value is written in the field "start time," and continuous bio-signals from this sensor will be recorded in the corresponding table, such as the ECG, $\mathrm{HR}$, or SPO2 table. The recording process will not stop until the switch is changed to " 0 ." The table formats of the ECG table, heart rate table, $\mathrm{SpO} 2$ table, and sensor are shown in Fig. 7.

The flowchart of Web-based application for the client-side user retrieving database is shown in Fig. 8. The monitoring process is described as follows: The electrocardiogram (ECG) or percentage saturation of haemoglobin $\left(\mathrm{SpO}_{2}\right)$ sensor measures the electrical signal of an ambulatory patient's heart continuously. The data are then transmitted to the router. When the router receives the data from the associated device, it sends the data to the associated PAN coordinator immediately. The PAN coordinator then sends the data to the UART connected with the UART of an industrial computer (IPC). A client-side program developed by Java takes the data from UART, processes these data, and then stores them in a remote database server by client-server socket connection.

\section{Results and Discussions}

When large data are transmitted to the database, data transmission performance should be considered. A comparison was made between a previous research and this work. Database access performance was evaluated by using two different database network connection methods.

\subsection{Comparison of data transmission performance.}

Usually, the maximum number of data of ECG signal transmitting to a database server can be limited by bandwidth of the connection network. Figure 9 shows the packet format for physiological data transmission from the gateway to the database server. It indicates that each piece of physiological information is four bytes. In practice, the required sampling rate of ECG waveforms is $250 \mathrm{~Hz}$. Therefore, to display verisimilar ECG waveforms, 250 records of physiological data must be transmitted from the gateway to the database server. This only requires $1 \mathrm{kbyte} / \mathrm{s}$ bandwidth. It is clear that as compared to the results of the previous research [7], less bandwidth is consumed, leading to the assumption that using low network connection speed is sufficient enough to achieve achieve data transmission. Another advantage is that the ECG waveform shown on the Web page is scalable vector graphics (SVG), which can be zoomed in and out with the same quality.

An experimental test using a real case has been conducted to evaluate the data transmission rate from the gateway to the database server for three different network connection speeds. Table 1 lists the various conditions for three different network connection speeds. The database server was in CTUST, which is located in Taichung and connected to TANET, with a connection speed of about 1,000 Mbps. Location A is in CTUST, which is located in the same laboratory as the database server, and is connected to TANET with a theoretical connection speed of 1,000 Mbps. Location B is in NTUT, which is located in Taipei, and is connected to TANET with a theoretical connection speed of $100 \mathrm{Mbps}$. Location $\mathrm{C}$ is in Taipei, which is $160 \mathrm{KM}$ (kilometer) from Taichung, and is connected to ISP APTG with a broadband network connection speed of 2M/256 Kbps (download/upload).

\begin{tabular}{|c|c|c|c|} 
Byte 1 & Byte 2 & Byte 3 & Byte 4 \\
\hline ID & Type & Data 1 & Data2 \\
\hline
\end{tabular}

Figure 9. The packet format for physiological signal transmission from the gateway to the database server.

Table 2 shows the experimental results for three different connection speeds and two database access methods. The experiment for each condition was repeated 30 times. Every time, ten thousand or 10000 records data were written continuously to the database server from the gateway, which is at a different location, to the server. The average transmission performance for various connection speeds and database access methods is plotted in Fig. 10. It can be seen that the transmission time of direct networking access is from $0.897 \mathrm{~ms}$ to $1.039 \mathrm{~ms}$ for three different test conditions. However, the transmission time of client-server access is from $1.030 \mathrm{~ms}$ to $23.878 \mathrm{~ms}$ for three different test conditions. The performance of 
client-server access is 5 to 20 times for direct networking access.

Table 1. Connection speed test at various conditions.

\begin{tabular}{crc}
\hline $\begin{array}{c}\text { Test } \\
\text { condition }\end{array}$ & $\begin{array}{c}\text { Connection } \\
\text { speed(bps) }\end{array}$ & Location \\
\hline $\mathrm{A}$ & $1,000 \mathrm{M}$ & Taichung(TANET) \\
\hline $\mathrm{B}$ & $100 \mathrm{M}$ & Taipei(TANET) \\
\hline $\mathrm{C}$ & $2 \mathrm{M} / 256 \mathrm{~K}$ & Taipei(ISP APTG) \\
\hline
\end{tabular}

Table 2. Transmission performance in different data access methods (ms).

\begin{tabular}{cccc}
\hline & $\mathrm{A}$ & $\mathrm{B}$ & $\mathrm{C}$ \\
\hline $\begin{array}{c}\text { Direct Networking } \\
\text { Access }\end{array}$ & 1.030 & 7.124 & 23.878 \\
\hline $\begin{array}{c}\text { Client-Server } \\
\text { Access }\end{array}$ & 0.897 & 0.951 & 1.039 \\
\hline
\end{tabular}

\subsection{Comparison of ECG waveform}

An original ECG waveform acquired from the sensor node is shown in Fig. 11(a), and the reconstructed waveform on the Web page is shown in Fig. 11(b). In this case, the percentage of rms difference (PRD) is $0 \%$, which is better than that of other transmission methods [7, 8]. It is clear that this study provides a reliable and accessible system for the end user (professional medical personnel or even the patients) to monitor a patient's physical condition.

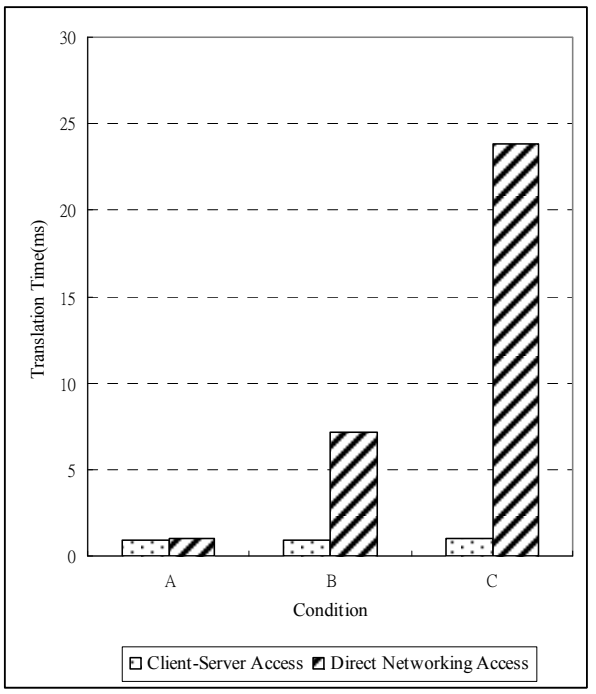

Figure 10. Experimental results of data transmission performance for different data access methods.

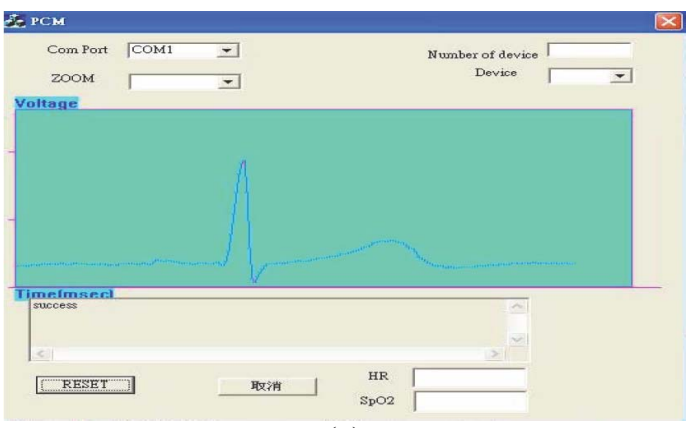

(a)

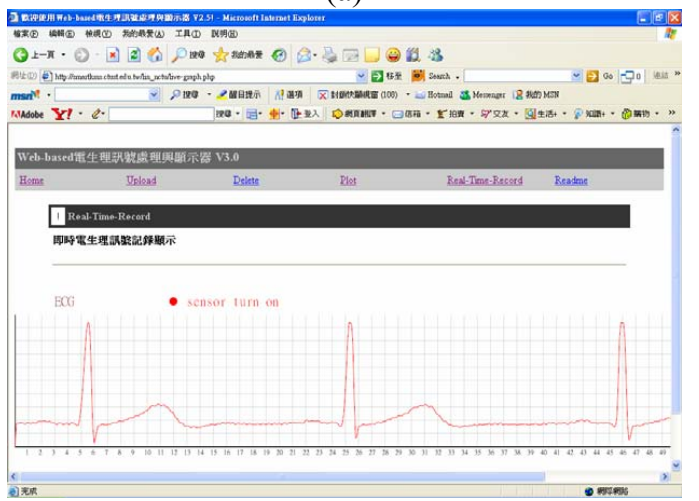

(b)

Figure 11. (a) Original ECG waveform acquired

from the sensor through RS-232. (b) The reconstructed ECG waveform on a Web-based interface acquired from the database server.

\section{Conclusions}

Generally, it is shown that the use of Web-based management interfaces for managing and querying sensor networks will become a trend in the future. This study uses the newest SVG and Ajax technologies to implement the Web-based bidirectional control interface with RIA concept. From the results, it is also revealed that the performance of the client-server access method is 5 to 20 times faster than that of the direct networking access method for three different network connection speeds. Most importantly, the novel technique used in this study allows users to easily control the remote sensor node through a Webbased interface via the Internet, a possibility which has not been explored before.

\section{Acknowledgment}

The authors would like to thank the National Science Council of the Republic of China for financially supporting this research under Contract No. NSC 95-2218-E-027-027- and 96-2218-E-027-001-. 


\section{References}

[1] "The World Health Report 2006", World Health Organization,

http://www.who.int/whr/2006/en/index.html.

[2] D. Faxon and C. Lenfant, "Timing Is Everything: Motivating Patients To Call 9-1-1 And Onset Of Acute Myocardial Infraction," Circulation, vol. 104, 2001, pp. 1210-1211.

[3] A.Tura, M. Badanai, D. Longo and L. Quareni, "A Medical Wearable Device With Wireless BluetoothBased Data Transmission," Measurement Sci. Rev., vol. 3, sec. 2, 2003, pp. 1-4.

[4] K.I. Hwang, J. In, N. Park and D.S Eom, "A Design And Implementation Of Wireless Sensor Gateway For Efficient Querying And Managing World Wide Web," IEEE Trans. Consum. Electron. vol. 49, no. 4, 2003, pp. 1090-1097.
[5] P. Ferrari, A. Flammini, D. Marioli, E. Sisinni, and A. Taroni, "A Bluetooth-Based Sensor Network With Web Interface," IEEE Transactions on Instrumentation and Measurement, vol. 54, no. 6, 2005, pp.2359-2363.

[6] K. J.Kerpez, T. E. Chapuran, R. C. Menendez, and S. S.Wagner, "Digital transmission over in-home coaxial wiring," IEEE Trans. Broadcasting, vol. 43, pp. 136144, June 1997.

[7] R.G. Lee, H.S. Chen, C.C. Lin, K.C. Chang, and J.H. Chen, "Home telecare system using cable television plants-An experimental field trial," IEEE Trans. Inf. Technol. Biomed., vol.4, no.1, pp.37-44, March 2000.

[8] M.A. Navakatikyan, C.J. Barrett, G.A. Head, and J.H. Ricketts, "A real-time algorithm for the quantification of blood pressure waveforms," IEEE Trans. Biomed. Eng., vol.49, no.7, pp.662-670, July 2002. 